\title{
Estimating and predicting corrections for empirical thermospheric models
}

\author{
E. Forootan ${ }^{\oplus}, 1,2$ S. Farzaneh, ${ }^{3}$ C. Lück ${ }^{4}$ and K. Vielberg ${ }^{4}$ \\ ${ }^{1}$ Institute of Physics and Meteorology (IPM), University of Hohenheim, Garbenstraße 30, 70599 Stuttgart, Germany. \\ E-mail: ehsan.forootan@uni-hohenheim.de \\ ${ }^{2}$ School of Earth and Ocean Sciences, Cardiff University, Cardiff CF103AT, UK \\ ${ }^{3}$ School of Surveying and Geospatial Engineering, College of Engineering, University of Tehran, Tehran 113654563, Iran \\ ${ }^{4}$ Institute of Geodesy and Geoinformation, University of Bonn, Nussallee 17, 53115 Bonn, Germany
}

Accepted 2019 March 29. Received 2019 March 18; in original form 2018 September 14

\begin{abstract}
SUMMAR Y
Quantifying spatial and temporal changes in thermospheric neutral density is important for various applications such as precise orbit determination, estimating mission lifetime and reentry prediction of Earth orbiting objects. It is also crucial for analysis of possible collisions between active satellite missions and space debris. Empirical models are frequently applied to estimate neutral densities at the position of satellites. But their accuracy is severely constrained by model simplifications and the sampling limitation of solar and geomagnetic indices used as inputs. In this study, we first estimate thermospheric neutral density by processing the high-accuracy accelerometer measurements on-board of the twin-satellite mission Gravity Recovery And Climate Experiment (GRACE). Daily density corrections (in terms of scales) are then computed for the commonly used NRLMSISE-00 empirical model. The importance of these daily scales is examined within an orbit determination practice. Finally, three data-driven prediction techniques based on Artificial Neural Network (ANN) are applied to forecast the daily density corrections for few days to months. Our numerical results indicate that GRACE derived scales are correlated with solar and geomagnetic indices and can improve the timing (from few hours to days) and magnitude of model simulations (up to 10-100 times) during high solar or geomagnetic activity when they usually perform poorly. We found that the Non-linear Autoregressive with Exogenous (External) Input (NARX) ANN technique performs well in predicting the corrections with an average fit of 0.8 or more in terms of squared correlation coefficients for time-scales of 7-90 days.
\end{abstract}

Key words: Thermospheric Neutral Density; Low-Earth-Orbit; Artificial Neural Network (ANN) Prediction; GRACE; Empirical Corrections.

\section{INTRODUCTION}

The Earth is surrounded by a thick layer of gas, known as the atmosphere. The total density of the Earth's atmosphere changes dynamically due to various reasons. A dominant part of such changes can be largely explained as cyclic variations, that is daily and seasonal, which are caused by changes in the relative positions of Earth and Sun, as well as the orientation of the Earth with respect to the Sun. These variations disproportionately influence heating of the atmosphere and consequently affect its density distribution. Vertically, thermospheric density decreases exponentially with altitude, and horizontally for altitudes between approximately 300 and $700 \mathrm{~km}$, the summer northern and southern latitude densities are greater than those of equator. For latitudes greater than $55^{\circ} \mathrm{N}$, the northern latitude to equatorial latitude density ratio is smallest during the day and increases at night. Density of regions close to the magnetic pole has been reported to be higher than that of the equator (see e.g. Qian \& Solomon 2012).

Changes in the solar and geomagnetic activity also have a significant impact on the (heating and) distribution of atmospheric neutral density, which cannot be easily described as a fundamental cyclic behaviour. Solar proxies are used to account for solar emissions, whereas the sunspot number and in particular the $F_{10.7}$ index (the solar $10.7 \mathrm{~cm}$ radio noise) is commonly used to represent solar activity (e.g. Bilitza 2000). Sometimes, irregular events take place on the Sun that cause unforeseen solar flares, as well as Coronal Mass Ejections (CME). These events have an enormous impact on the Earth's magnetic field, too. The intensity of geomagnetic variations, which is expressed by the $K_{p}$ or $A_{p}$ index (Mayaud 1980) controls the equilibrium of the neutral and ionized thermosphere. In addition, ionospheric activity affects the energy content of atmosphere and has an influence on its dynamics and 
chemical composition. For example, the ionospheric plasma convection at high latitudes transfers energy and momentum, influences Joule heating and accelerates the movements of neutral and ionized items. The thermospheric neutral wind moves the plasma and generates electric fields, which also affect the equilibrium of the neutral and ionized parts of the atmosphere (see, e.g. Qian \& Solomon 2012; Xu et al. 2015).

The state of the atmosphere is very important for many satellite related applications such as precise orbit determination, planning mission lifetime, re-entry prediction, and collision analysis. Atmospheric density at satellite level altitudes will determine the drag acting on the surface of satellites, and therefore, the speed at which they decelerate. Considering the altitude-dependent nature of changes in thermospheric neutral density, it is expected that within the altitude of Low-Earth-Orbit (LEO) satellites, that is, between 150 and $\sim 600$ or $1000 \mathrm{~km}$, the impact of drag on satellite orbit is considerable, and above $\sim 1000 \mathrm{~km}$, this force decreases. Within this altitude range, thermosphere and ionosphere are highly dynamic. Therefore, in recent decades, both empirical and physical models of the atmosphere have been considerably improved (see an overview of models used in space weather applications in, e.g. Belehaki et al. 2009).

The Mass Spectrometer and Incoherent Scatter (MSIS) empirical models of the neutral atmosphere (Picone et al. 2002) have been developed since 1977, and the last two versions MSISE90 and NRLMSISE-00 contain an extensive data set including remote sensing and in situ measurements. Other models such as the series of Jacchia models, for example Jacchia (1970) and that of Bowman et al. (2008), have been primarily built upon large orbital drag databases. The empirical models are parametrized in terms of solar and geomagnetic (storm) indices that are daily, 3-hourly, and hourly at their highest resolution. The Drag Temperature Model (DTM, Bruinsma et al. 2003), a semi-empirical model, and other physical and data assimilative models (e.g. Thermosphere Ionosphere Electrodynamic General Circulation Model (TIE-GCM Roble \& Ridley 1994, www.hao.ucar.edu/modeling/tgcm/), and the High Accuracy Satellite Drag Model (HASDM, Storz et al. 2005)) attempt to include more dynamic processes of the thermosphere-ionosphere and/or satellite driven auxiliary data with the final goal of providing neutral density and temperature simulations closer (than empirical models) to reality.

Accelerometers on-board satellite gravimetry missions, such as CHAMP (CHAllenging Minisatellite Payload, Reigber et al. 2002), GRACE (Gravity Recovery And Climate Experiment, Tapley et al. 2004), GOCE (Gravity field and steady-state Ocean Circulation Explorer, Albertella et al. 2002) and the European Space Agency (ESA)'s Swarm mission measure non-gravitational forces, which can be used to improve the understanding of the thermospheric density and winds. Previous studies have already provided valuable information about the techniques used to retrieve atmospheric density from accelerometer observations. For example, Bruinsma \& Biancale (2003a) and Bruinsma et al. (2004) addressed the estimation of total neutral density from CHAMP accelerometer observations. Sutton (2008) and Doornbos (2011) provided algorithms to estimate density, and crosswind information from CHAMP and GRACE. Application of GOCE observations for density and wind estimations is discussed, for example, in Bruinsma (2013).

In fact, an accurate conversion of on-board accelerometer observations to thermospheric neutral density depends on properly (i) calibrating the acceleration measurements, (ii) modelling of the aerodynamic of satellites (including the geometry of plates and their orientation, as well as their materials), (iii) estimating the drag coefficient ( ' $\vec{C}_{D}$ ' and its possible temporal variations) and (iv) reducing undesired non-gravitational forces due to the solar and Earth radiation pressure. In particular, the processing of (i) directly scales density estimation and (ii) defines the interaction of satellite with the atmosphere. The drag coefficient in (iii) varies considerably, for example, a value between 2 and 4 has been estimated for $\vec{C}_{D}$ (under a hypervelocity and continuum flow assumption) that scales the density estimation from accelerometer observations (Vallado \& Finkleman 2014, see also Section 2). Assumptions about the energy emitted from the Sun and Earth affect the estimations in (iii), therefore should be taken into account (see, e.g., Mehta et al. 2017a).

In this study, (A) we apply the thermospheric neutral density estimation procedure, recently published in Vielberg et al. (2018), to estimate thermospheric neutral density using GRACE accelerometer observations. (B) Error sources influencing satellite derived density estimations (associated with the above step i to iii) are discussed. Next, (C) daily scales are computed using the GRACE derived along-track neutral density estimates and densities derived from empirical models. These scales are estimated for the NRLMSISE00 model, which are interpreted here as empirical corrections for this model. (D) The effect of these corrections is then evaluated for a range of orbits at altitudes of 200, 400 and $600 \mathrm{~km}$. Once we proved that the estimated daily scales are useful, (E) various data-driven prediction techniques are tested to predict corrections for empirical models when no GRACE data are available. These prediction techniques are formulated based on non-linear Artificial Neural Network (see other applications in, e.g. Choury et al. 2013; Pérez et al. 2014), which include Non-linear AutoRegressive (NAR), Non-linear Autoregressive with Exogenous (External) Input (NARX), and Non-Linear Neural Network Regression (NLNNR). All these techniques are discussed in Section 2.3.

State-of-the-art calibration and/or data assimilation techniques have been applied in previous studies (e.g., Fuller-Rowell et al. 2004; Codrescu et al. 2004, 2018; Minter et al. 2004; Matsuo et al. 2012, 2013; Morozov et al. 2013; Murray et al. 2015; Sutton 2018) to integrate model simulations with data such as temperature and neutral density estimates derived from satellite observations. These methods are, however, computationally expensive, and their accuracy in providing now-casts and forecasts of neutral density is also limited as discussed in Mehta \& Linares (2018) and Sutton (2018). This has motivated us to formulate a simple empirical correction and prediction system using efficient ANN models. We will show that this combination can provide 1 week to 3 months forecasts with acceptable range of uncertainties. However, after 3 months, the magnitude of predictions' uncertainty will equal the magnitude of the signal itself, that is up to 10 times bigger than the observations' uncertainties. Besides, the main assumption in our prediction is that the forecast of solar and geomagnetic indices is available with the same uncertainty of the observed indices. Once one considers higher magnitude for the uncertainty of indices, the seasonal predictions of our corrections might be interpreted with caution. From available models, we selected NRLMSISE-00 to present the numerical results. Applying other LEO observations such as those of CHAMP and GOCE or selecting other empirical models does not harm the main conclusion of the study, but the details of possible biases in different models might be to some extent changed. 


\section{DENSITY ESTIMATION FROM ON-BOARD ACCELEROMETER MEASUREMENTS}

The acceleration measurements on-board GRACE satellites $\vec{a}_{\text {obs }}$ reflect the predominant forces due to non-gravitational forces including aerodynamics $\vec{a}_{\text {aero }}$, solar radiation pressure $\vec{a}_{\text {SRP }}$, and the Earth radiation pressure $\vec{a}_{\text {ERP }}$ as

$\vec{a}_{\mathrm{obs}}=\vec{a}_{\mathrm{aero}}+\vec{a}_{\mathrm{SRP}}+\vec{a}_{\mathrm{ERP}}$.

The acceleration caused by aerodynamics $\vec{a}_{\text {aero }}$ including drag and lift can be described as

$\vec{a}_{\text {aero }}=\frac{1}{2} \vec{C}_{\mathrm{a}} \frac{A}{m} \rho\left|\vec{v}_{\text {rel }}\right|^{2}$,

where $\rho$ is the thermospheric neutral density surrounding the satellite, $\vec{C}_{\mathrm{a}}$ is the dimensionless force coefficient vector, the reference area $A$ is the projection of the area of satellite onto the current flow direction, $m$ is the mass of satellite and $\vec{v}_{\text {rel }}$ represents the velocity of the satellite with respect to the surrounding atmosphere. By combining eqs (1) and (2), the neutral density of thermosphere $(\rho)$ can be computed by

$\rho=\frac{2 m\left(\vec{a}_{\mathrm{obs}}-\vec{a}_{\mathrm{SRP}}-\vec{a}_{\mathrm{ERP}}\right) \cdot \vec{x}}{A \vec{C}_{\mathrm{a}}\left|\vec{v}_{\mathrm{rel}}\right|^{2} \cdot \vec{x}}$.

The drag force acts (on the surface of satellite) to the opposite direction of velocity that is why an inner product (multiplying by $\vec{x}=[1,0,0]^{T}$ in the satellite body-fixed reference frame) is applied in eq. (3). In thermospheric density estimation, calibration of accelerometer data (reflected in $\vec{a}_{\text {obs }}$ ), aerodynamic of the satellite and its orientation in the orbit are important. In this study, calibration of accelerometer observations is performed following Vielberg et al. (2018), and the aerodynamic acceleration is modelled following Doornbos (2011) using 8 plates (as in Bettadpur 2012).

Solar Radiation Pressure (SRP, $\vec{a}_{\mathrm{SRP}}$ ) results from the impact of electromagnetic radiation, emitted from the Sun, on a moving satellite, and Earth Radiation Pressure (ERP, $\vec{a}_{\mathrm{ERP}}$ in eq. (3)) consists of accelerations caused by albedo ( $\left.\vec{a}_{\text {albedo }}\right)$, as well as thermal emission of the Earth $\left(\vec{a}_{\mathrm{IR}}\right)$. Here, SRP and ERP are estimated for the GRACE macromodel following the formulations presented in Vielberg et al. (2018). For the ERP, the satellite data from the latest version of the Cloud and the Earth's Radiant Energy System (CERES) EBAF Ed4.0 (Loeb et al. 2018) obtained from the NASA Langley Research Center CERES ordering tool (http://ceres.larc.nasa.gov/, accessed: 5 November 2017) are used.

\subsection{Uncertainties due to the mismodelling of aerodynamic-material properties}

Uncertainty estimation for the accelerometer derived neutral density profiles is rather complicated because many variables of eq. (3) are interrelated. Here, we assess the uncertainty using a variance propagation method (see eq. 4). The variance propagation equation is derived here as:

$\sigma_{\rho}=\sqrt{\vec{F} \Sigma^{-1} \vec{F}^{\top}}$,

where $\vec{F}$ is a vector with all partial derivatives

$\vec{F}=\left[\frac{\partial \rho}{\partial m} \frac{\partial \rho}{\partial a_{\mathrm{obs}, \mathrm{x}}} \frac{\partial \rho}{\partial a_{\mathrm{SRP}, \mathrm{x}}} \frac{\partial \rho}{\partial a_{\mathrm{ERP}, \mathrm{x}}} \frac{\partial \rho}{\partial v_{\mathrm{rel}, \mathrm{x}}} \frac{\partial \rho}{\partial C_{\mathrm{a}, \mathrm{x}}}\right]$,

and $\Sigma$ is the matrix that contains the covariance of errors as

$\Sigma=\operatorname{diag}\left(\sigma_{m}^{2}, \sigma_{a_{\mathrm{obs}, \mathrm{x}}}^{2}, \sigma_{a_{\mathrm{SRP}, \mathrm{x}}}^{2}, \sigma_{a_{\mathrm{ERP}, \mathrm{x}}}^{2}, \sigma_{v_{\mathrm{rel}, \mathrm{x}}}^{2}, \sigma_{C_{\mathrm{a}, \mathrm{x}}}^{2}\right)$.
Table 1. Uncertainties of the variables that affect the computation of thermospheric neutral density in eq. (3). The index $x$ denotes that the uncertainty is only valid in the along-track direction.

\begin{tabular}{|c|c|}
\hline Component & Uncertainty \\
\hline$\sigma_{m}$ & $0.5 \mathrm{~kg}$ \\
\hline$\sigma_{a_{\mathrm{obs}, \mathrm{x}}}$ & $10^{-8} \mathrm{~m} \mathrm{~s}^{-2}$ \\
\hline$\sigma_{a_{\mathrm{SRP}, \mathrm{x}}}$ & $10^{-9} \mathrm{~m} \mathrm{~s}^{-2}$ \\
\hline$\sigma_{a_{\mathrm{ERP}, \mathrm{x}}}$ & $10^{-11} \mathrm{~m} \mathrm{~s}^{-2}$ \\
\hline$\sigma_{a_{v_{\mathrm{rel}, \mathrm{x}}, \mathrm{x}}}$ & $100 \mathrm{~m} \mathrm{~s}^{-1}$ \\
\hline$\sigma_{C_{\mathrm{a}, \mathrm{x}}}$ & 0.1 \\
\hline
\end{tabular}

For the variance propagation, errors (from literature) are used and propagated to estimate the uncertainty of thermospheric neutral density $(\rho)$. The error term for the reference area $A$ is not considered here as it also appears in the force coefficient vector $\left(\vec{C}_{\mathrm{a}}\right)$. Therefore, its impact on the computation of $\rho$ cancels out. We should also note that Flury et al. (2008) assumed an error magnitude of $10^{-10} \frac{\mathrm{m}}{\mathrm{s}^{2}}$ for accelerometer observations. Here, we assume a more realistic value of $10^{-8} \frac{\mathrm{m}}{\mathrm{s}^{2}}$ to reflect the biases as explained in Vielberg et al. (2018). In fact, uncertainty of the accelerometer observations fluctuates during the mission's lifetime, which is not taken into account in this study. Following Doornbos et al. (2009), an error of $100 \frac{\mathrm{m}}{\mathrm{s}}$ is considered for the relative velocity. Uncertainties of the variables that are used in the error propagation procedure are reported in Table 1. We also validated the results of eq. (4) with the output of a Monte Carlo experiment, in which the errors of Table 1 (all together) were considered. The results (not shown here) are found to be in agreement with the error propagation using the variance propagation approach.

\subsection{Daily scales as empirical corrections}

Corrections of empirical density models are computed as a fraction of the along-track thermospheric neutral densities obtained from GRACE (eq. 3) and the corresponding neutral density from empirical models, that is here NRLMSISE-00. For this, (1) thermospheric neutral density is computed using GRACE observations within a day; (2) NRLMSISE-00 model is run to generate density values at the same time and location as the GRACE in (1) and (3) GRACE derived density estimations of (1) is divided by those obtained from NRLMSISE-00 in (2) to calculate the unit-less values, which is referred to as 'scales'

$d=\rho_{\mathrm{GRACE}} / \rho_{\text {model }}$.

These scales are computed at every 10 second intervals, that is at each given time and location of the satellite orbit, which are then expressed in terms of the spherical harmonic coefficients over a full day to derive a smooth field of global scales that does not dramatically change the structure of the thermospheric model. Thus, this scale field can be interpreted as a daily mean to modify the model-derived densities.

The daily scale fields $(d)$ are expanded in terms of spherical harmonics coefficients as

$$
\left.\begin{array}{l}
C_{n m} \\
S_{n m}
\end{array}\right\}=\int_{\sigma} \mathbf{d} \bar{P}_{n m}(\cos \theta)\left\{\begin{array}{c}
\cos m \lambda \\
\sin m \lambda
\end{array}\right\} \mathrm{d} \sigma,
$$

where $\bar{P}_{n m}$ are the fully normalized associated Legendre polynomials, $\theta$ and $\lambda$ are the colatitude and longitude, and $\mathrm{d} \sigma$ is the surface element. We solve this integral numerically following the approach in Forootan et al. (2013) and limit the maximum degree and order to 10 . Finally, the scale fields can be obtained by synthesizing 


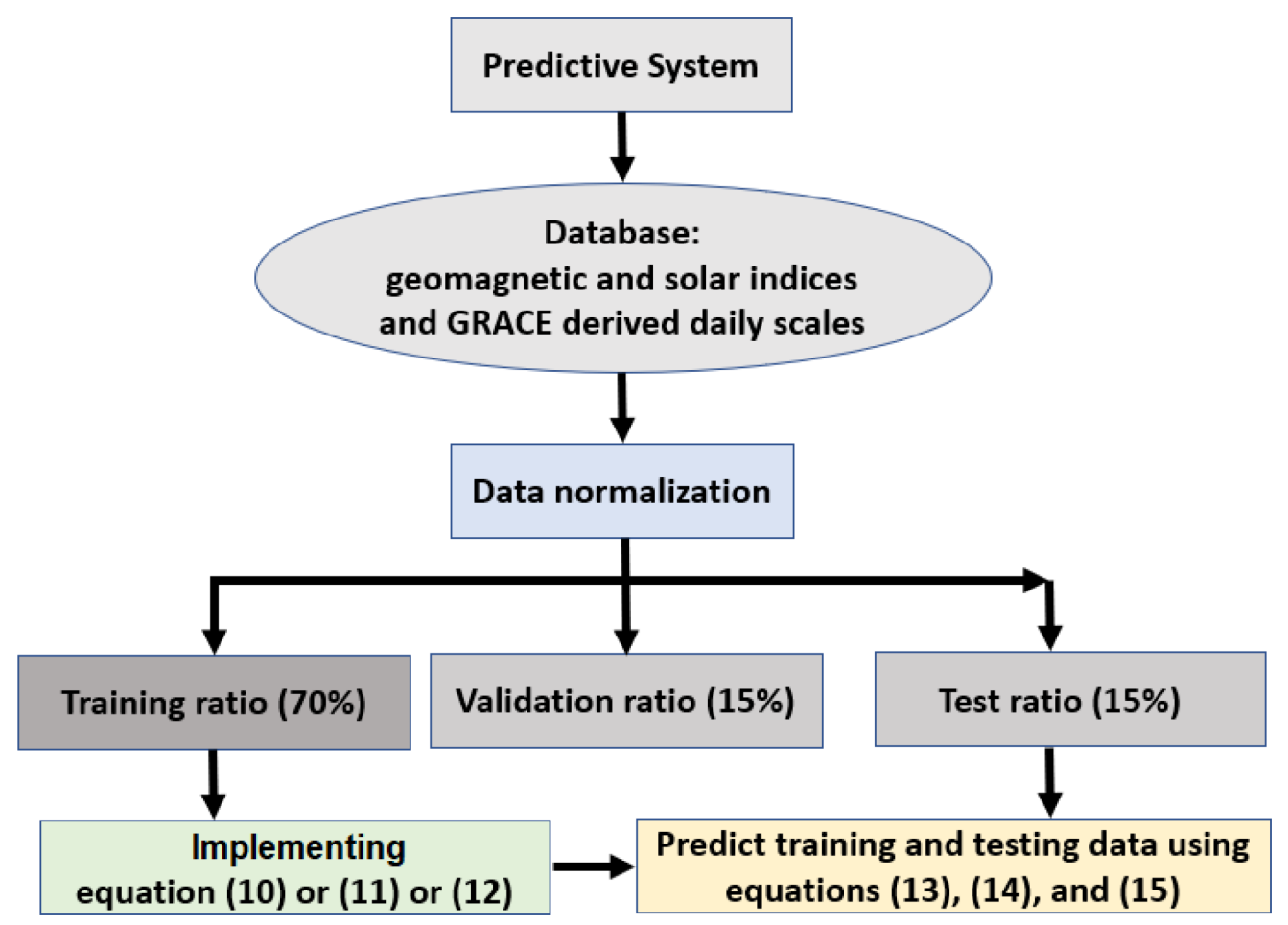

Figure 1. Flowchart of the ANN model identification applied in this study.

the estimated spherical harmonic coefficients on a desired location by

$\hat{d}(\theta, \lambda)=\frac{1}{4 \pi} \sum_{n=1}^{10} \sum_{m=0}^{N} \bar{P}_{n m}(\cos \theta)\left(C_{n m} \cos m \lambda+S_{n m} \sin m \lambda\right)(9)$

An example of the updated global thermospheric neutral density of 1 February 2004, 18:00 hr is shown in the Appendix A.

\subsection{Artificial neural network (ANN) for prediction}

This section provides an overview of the (data-driven) ANN techniques used in this study to predict daily empirical correction (scales). Generally speaking, data driven processes are governed by systems of linear or non-linear equations, which describe the relationship between the current and previous values of the system's output and the values of inputs. In this study, outputs are predicted time-variable scales of few days to few months. Our inputs are the computed scales in eq. (9), and in some cases, we also use geomagnetic and solar radiation indices as inputs. All input data are generated up-to-date in order for the ANN model to continuously generate the outputs.

While there are many prediction models available, we specifically use the ANN techniques because they are able to deal with the non-linear changes of thermospheric density due to solar and geomagnetic changes. We tested linear techniques such autoregression and autoregressive with exogenous input following the formulation in Forootan et al. (2014) and the results suggested that the accuracy of predictions might not be acceptable when sudden anomalies appear after a smooth evolution of density changes (results are not shown). In general, an ANN method consists of 'layers', which are made of a set of interconnected nodes, known as 'neurons' that contain 'output functions' (activation functions). These functions make a linkage between the neurons and the numerical weights that are used to combine the inputs and simulate the outputs. Independent variables, as the input data, enter into the ANN via the 'first (input) layer', and dependent variables are obtained from the 'last (output) layer'. The 'hidden layer' is to transform the inputs into something that the output layer can use. By choosing various non-linear functions, different arrangement of the neurons, and layers, non-linear response to the input variables would be captured. Therefore, adaptivity is an important feature of the ANNs (Chakraverty \& Mall 2017).

Several feed-forward algorithms exist, which can be used to account for non-linear relationships. Each of these algorithms has their own advantages and disadvantages. Here, we follow Chen \& Billings (1991) to implement a radial base function network, for which radially symmetric responses can be derived for each node. The advantage of this approach is that it applies least squares adjustment plus clustering techniques, therefore, it can be considered as a direct extension of already tested linear regression techniques, for example, those methods presented in Forootan et al. (2014).

\subsubsection{Categories of mathematical models to implement non-linear ANNS}

Models examined here include Non-linear AutoRegressive (NAR), Non-linear Autoregressive with Exogenous (External) Input (NARX) and Non-Linear Neural Network Regression (NLNNR), which are summarized below. These three techniques are selected to test how changing the combination of inputs might help the prediction of scales. In summary, NAR uses only the information in the time series of scales, NARX uses geomagnetic and solar radiation indices in addition to the scales as inputs, while NLNNR uses only solar and geomagnetic indices to train the prediction.

NAR is formulated in eq. (10) to relate the scale at time $t(y(t))$ to the past consecutive $d$ days $(t-d \ldots t-1)$. In other words, the value of scales at time $t$ is predicted using its ' $d$ ' past values. Therefore, 
(A) 01.01.2004

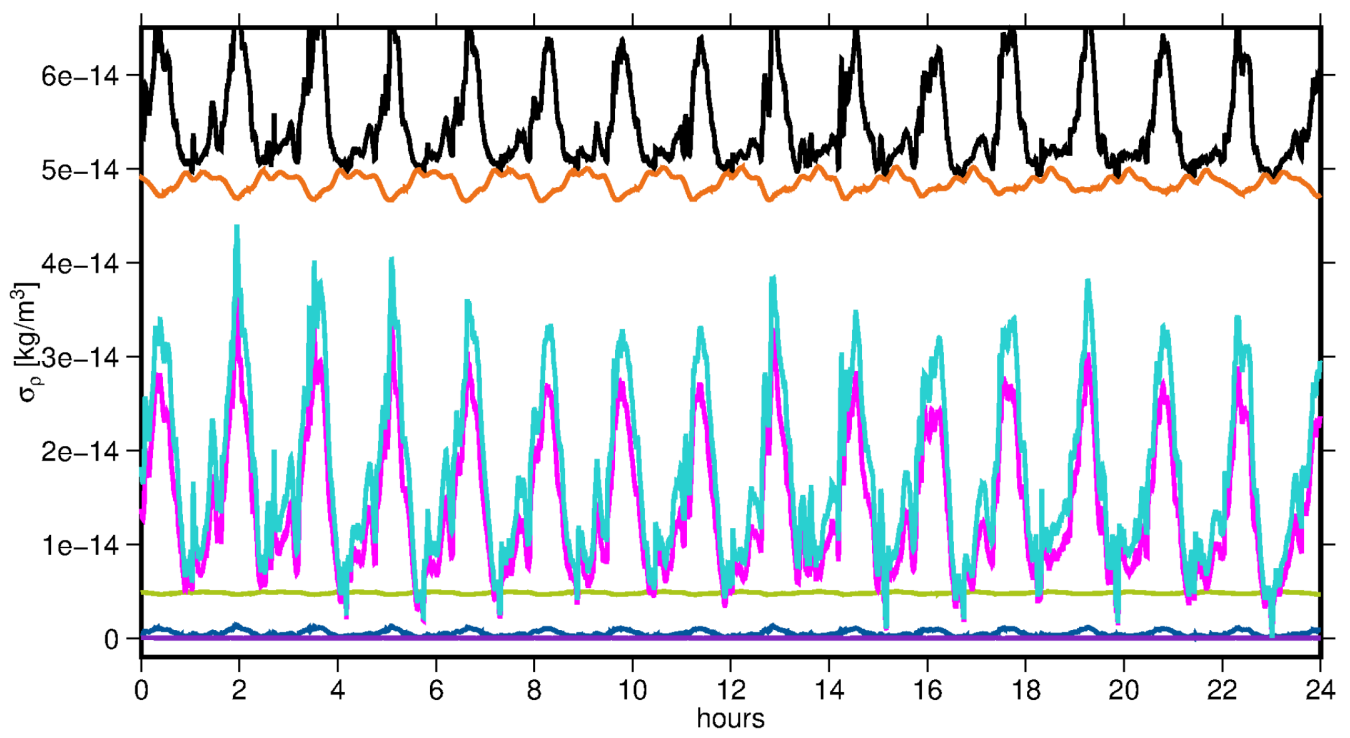

(b) 01.01 .2014

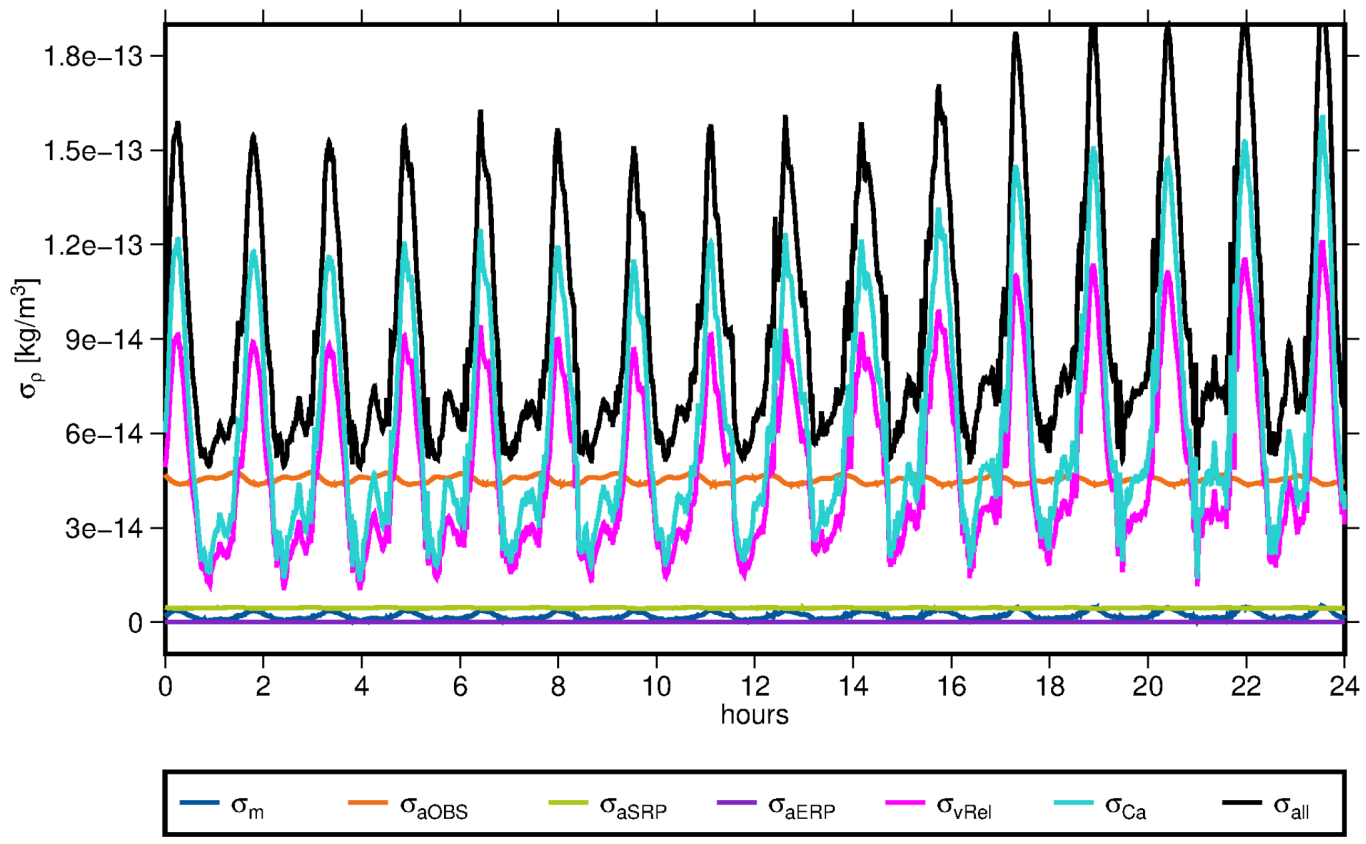

Figure 2. Effect of different sources of uncertainty on the density of GRACE A on (a) 1 January 2004 and (b) 1 January 2014. The density itself is in the order of $10^{-12} \mathrm{~kg} \mathrm{~m}^{-3}$ (not shown here).

NAR is defined as

$y(t)=f(y(t-1), y(t-2), \ldots, y(t-d))+\epsilon$,

where the activation function $(f)$ is estimated using the multilayer perception (MLP, Kisi 2004), and $\epsilon$ contains the residuals. To keep the implementations consistent and comparable, this selection of MLP is kept unchanged for the NARX and NLNNR methods.

NARX is a recurrent dynamic ANN, with feedback connections encompassing multiple layers. It can be efficiently used for modelling non-stationary and non-linear time-series. Mathematically, the input-output representation of non-linear discrete time-series in
NARX network is governed by the following equation:

$$
\begin{aligned}
y(t)= & f(y(t-1), y(t-2), \ldots, y(t-d), x(t-1), \\
& \left.x(t-2), \ldots, x\left(t-d^{\prime}\right)\right)+\epsilon,
\end{aligned}
$$

where $y(t)$ represents the values of dependent output, and $x$ represents the externally determined variable(s) that influence(s) the output values $(y)$. In our case, we select $y$ to be empirical scales and $x$ to be geomagnetic and solar radiation indices $\left(K_{p}\right.$ and $A_{p}$, respectively). 
(a) 2004

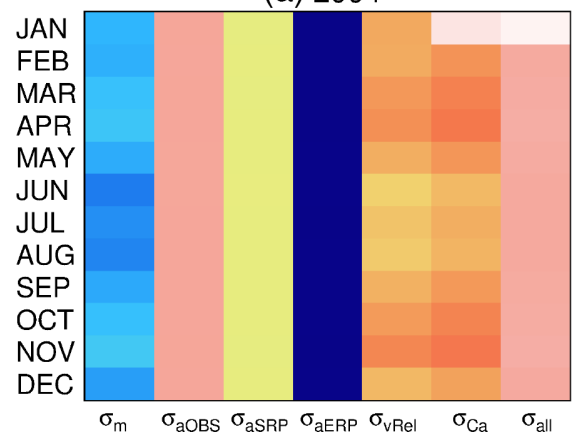

(b) 2014

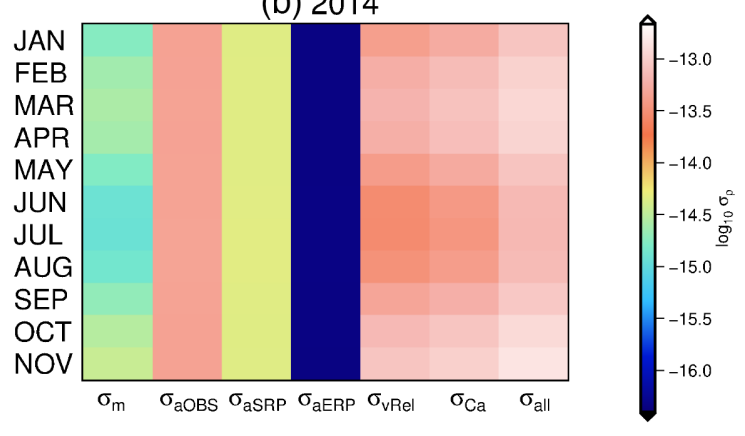

Figure 3. Effect of different sources of uncertainty on the density of GRACE A as monthly means on (a) 2004 and (b) 2014.

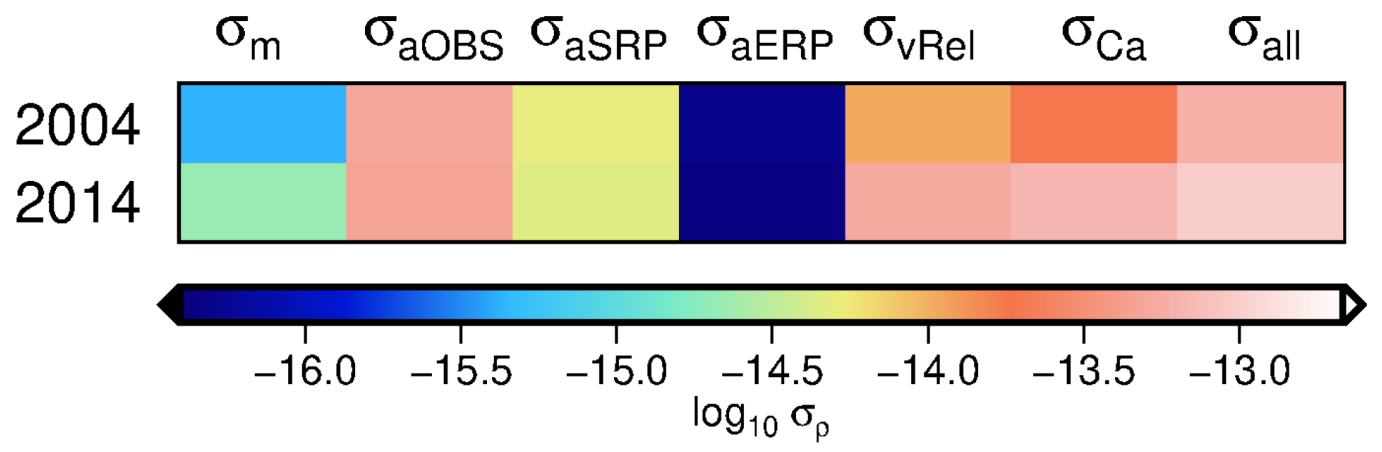

Figure 4. Effect of different sources of uncertainty on the density of GRACE A as yearly means on (top-row) 2004 and (bottom-row) 2014.

Table 2. SNR values, expressed as $\frac{\rho}{\sigma_{\rho_{\mathrm{all}}}}$ for 2004 and 2014, where $\rho$ is density and $\sigma_{\rho_{\text {all }}}$ represents the overall uncertainty.

\begin{tabular}{lcc}
\hline & \multicolumn{1}{c}{2004} & 2014 \\
\hline SNR $>1$ & 94.0 per cent & 100.0 percent \\
SNR $>2$ & 89.2 percent & 100.0 percent \\
SNR $>5$ & 63.8 percent & 99.8 percent \\
SNR $>10$ & 28.0 percent & 97.4 percent \\
SNR $>20$ & 0.3 percent & 54.8 percent \\
\hline
\end{tabular}

NLNNR is defined here as a mathematical relationship between inputs (predictors) and outputs (predictants). The difference between this approach and NARX is that the values of scales are not used in the tuning of the neural network. Therefore, the formulation is simplified as

$y(t)=f(x(t-1), x(t-2), \ldots, x(t-d))+\epsilon$,

where $y(t)$ represents the scales to be predicted and $x$ to be geomagnetic and solar radiation indices.

The above three formulations of NAR, NARX and NLNNR provide us the opportunity to use either only the previous values of outputs (eq. 10), values of outputs and a set of indicators (eq. 11) and only indicators (eq. 12) for predictions, respectively. Therefore, one will have more flexibility to design the prediction considering the availability of input data and indicators.

\subsubsection{Metrics to optimize ANNS}

Several metrics are used to validate the outputs of various ANN implementations, which include the mean squared error (MSE, eq. 13), signal-to-noise ratio (SNR, eq. 14), and the squared correlation coefficient $\left(R^{2}\right.$, eq. 15$)$ between the outputs and targets of ANNs.
Table 3. Initial conditions for three dynamic orbit propagation experiments. Values are reported in the Earth Centred Inertial (ECI) system.

\begin{tabular}{lc}
\hline Parameters & Values \\
\hline Date (DAY, MONTH, YEAR) & $04-30032004$ \\
Time (Hour, Min, Sec) & 000005 \\
Position (m) for altitude $200 \mathrm{~km}$ & $x=6574062.515$ \\
& $y=1837.650$ \\
& $z=38967.280$ \\
Velocity $\left(\mathrm{m} \mathrm{s}^{-1}\right.$ ) for altitude $200 \mathrm{~km}$ & $v_{x}=-46.112$ \\
& $v_{y}=367.525$ \\
& $v_{z}=7793.365$ \\
Position (m) for altitude $400 \mathrm{~km}$ & $x=6773269.196$ \\
& $y=1810.427$ \\
Velocity (m s ${ }^{-1}$ ) for altitude $400 \mathrm{~km}$ & $z=38390.024$ \\
& $v_{x}=-43.440$ \\
Position (m) for altitude $600 \mathrm{~km}$ & $v_{y}=362.081$ \\
& $v_{z}=7677.922$ \\
& $x=6972475.313$ \\
Velocity (m s ${ }^{-1}$ ) for altitude $600 \mathrm{~km}$ & $y=1784.379$ \\
& $z=37837.684$ \\
Semi-major axis (m) & $v_{x}=-40.993$ \\
Eccentricity & $v_{y}=356.872$ \\
Inclination (o) & $v_{z}=7567.462$ \\
Right-Ascension of the ascending node (o) & 6827998.673 \\
Argument of perigee (o) & 0.004 \\
True anomaly (o) & 87.3 \\
\hline & 0 \\
& 0.2106 \\
\hline
\end{tabular}

These can, respectively, be computed as

$\mathrm{MSE}=\frac{1}{\mathrm{~N}} \sum_{\mathrm{i}=1}^{\mathrm{N}}\left(\mathrm{y}_{\mathrm{i}}-\hat{\mathrm{y}}_{\mathrm{i}}\right)^{2}$, 


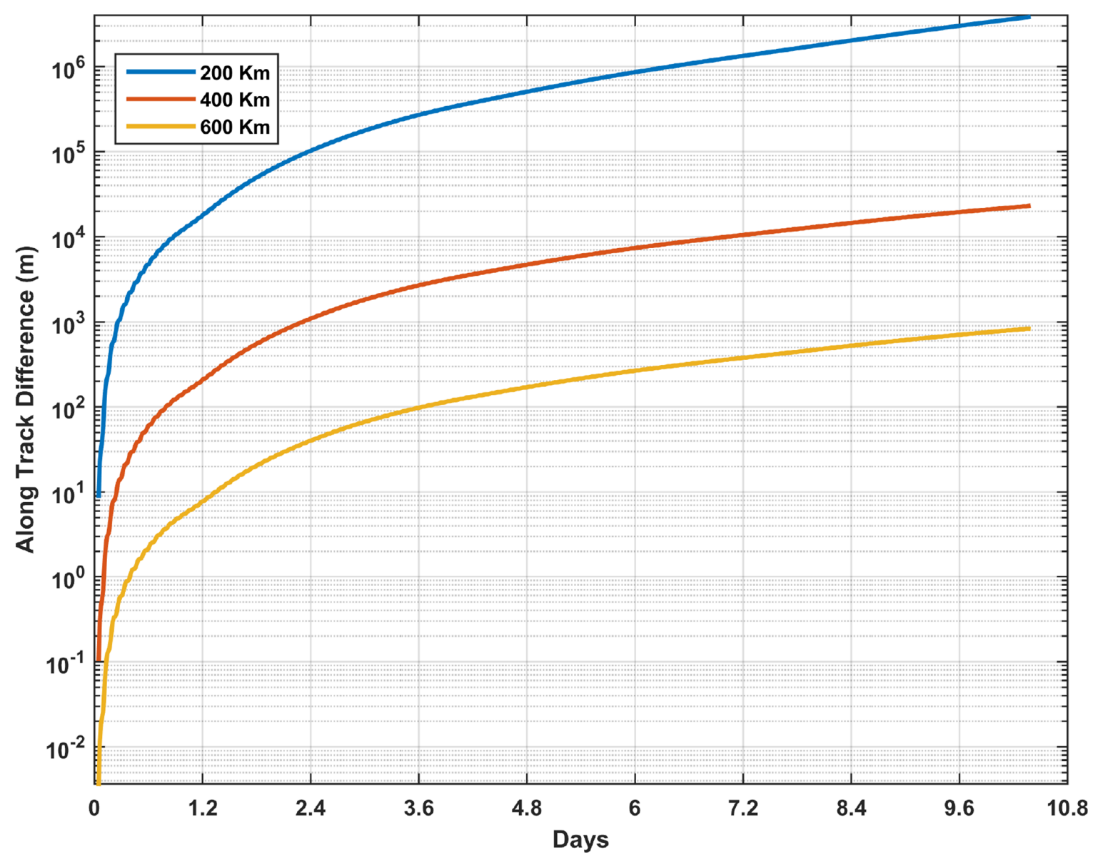

Figure 5. Along-track differences of the orbital positions for $10 \mathrm{~d}$ from 4 March 2004. Orbits are predicted using original and scaled thermospheric neutral density outputs of NRLMSISE-00.
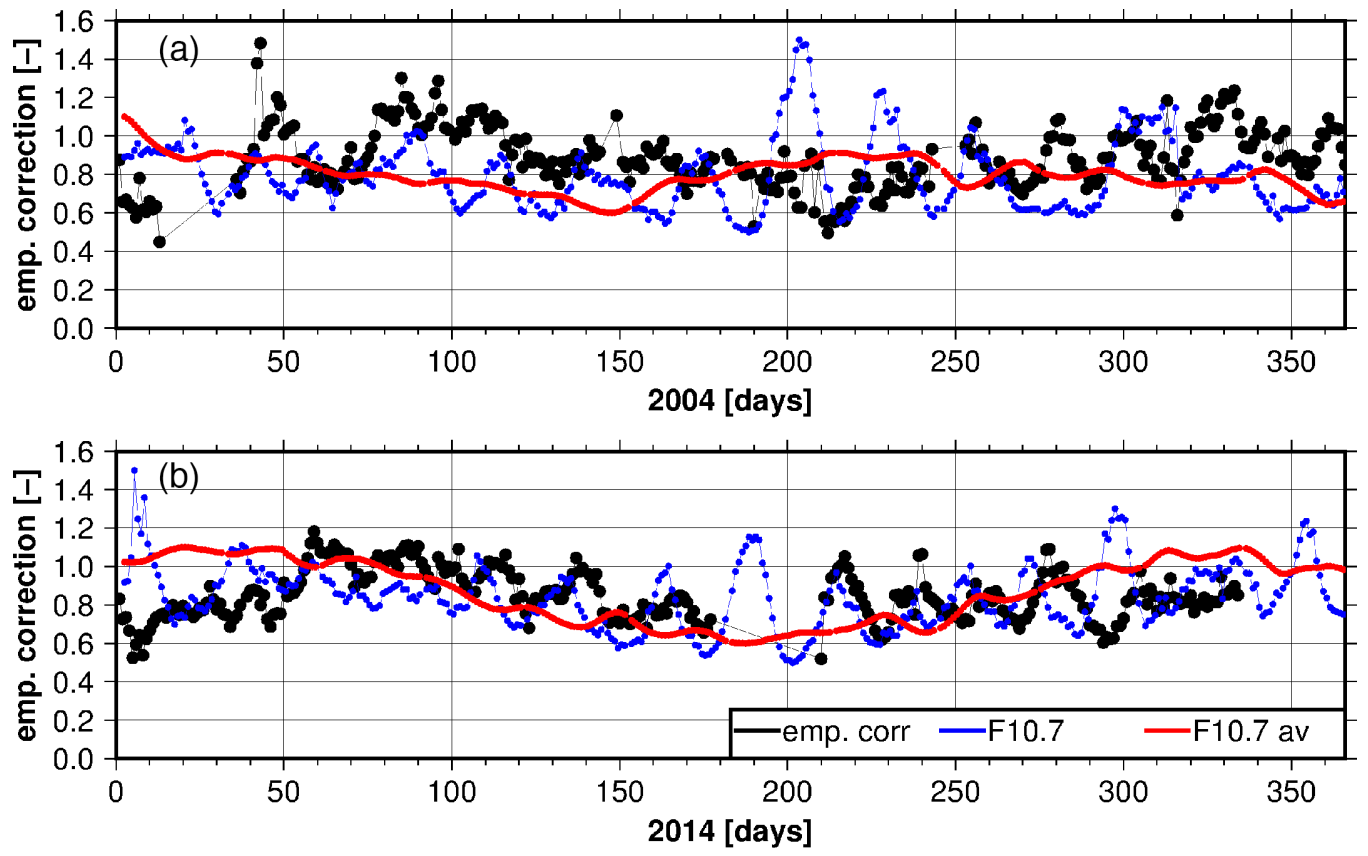

Figure 6. Daily mean of empirical density corrections for NRLMSISE-00 in 2004 (a) and 2014 (b).

$\mathrm{SNR}=10 \log _{10}^{\frac{(\max (\mathrm{y}))^{2} \mathrm{~N}}{\sum_{\mathrm{i}=1}^{\mathrm{N}\left(\mathrm{yi}_{\mathrm{i}}-\hat{\mathrm{y}}_{\mathrm{i}}\right)^{2}}}}$,

and

$\mathrm{R}^{2}=1-\left(\frac{\sum\left(\hat{\mathrm{y}}-\mathrm{y}_{\mathrm{i}}\right)^{2}}{\sum\left(\mathrm{y}_{\mathrm{i}}-\overline{\mathrm{y}}_{\mathrm{t}}\right)^{2}}\right)$,

where $N$ is the total number of data, $\mathbf{y}_{i}$ is an array of target values, $\hat{\mathbf{y}}_{i}$ is the corresponding estimated values and $\overline{\mathbf{y}}_{t}$ is the mean value of the training set.
To construct efficient ANNs, several factors are tested here, which include: the number of hidden layers, length of delay, and the training period. This is implemented empirically by changing the number of delays from 1 to 15 , and the training length from 40 to 90 , and the delay length of 5 to $90 \mathrm{~d}$. Training is implemented using 70 per cent of available time series in 2003 ans 2004 and the remaining 30 percent is used for validation and test. To train ANNs and compute the regression coefficients (b and c), the Levenberg-Marquardt optimization technique (Chan et al. 2012) is used. The best ANN structure is selected to be the one with the best 


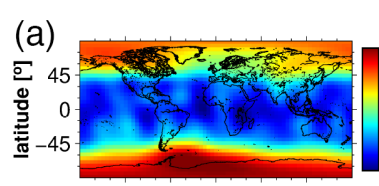

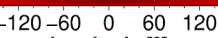
longitude $\left[{ }^{\circ}\right]$
总 (b)

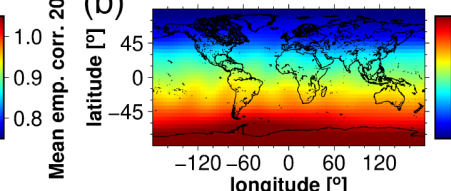

longitude $\left[{ }^{\circ}\right]$ (c)

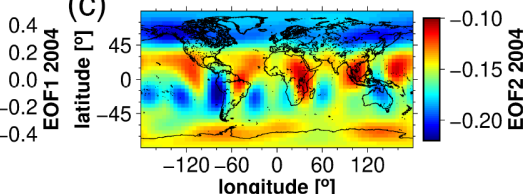

(d)

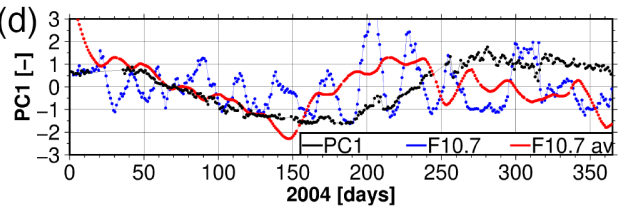

(e)

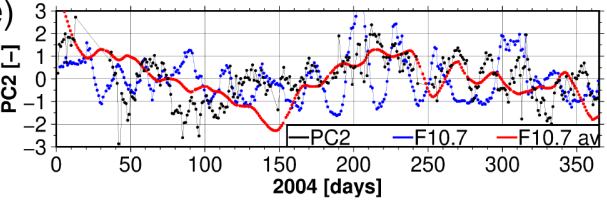

Corrected Density $(s, t)=(A(s)+B(s) D(t)+C(s) E(t)) *$ Model Density $(s, t)$

Figure 7. Temporal mean (a), and PCA derived spatial (b, c) and temporal (d, e) variability of empirical density corrections in 2004.

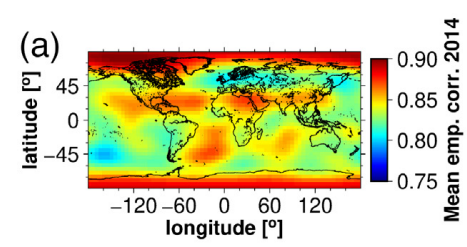

(d)

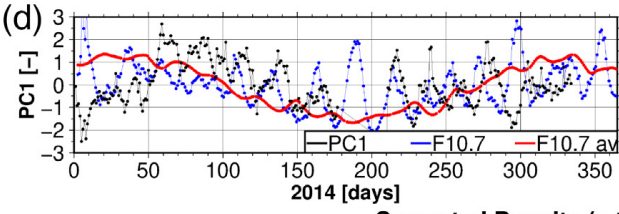

(b)

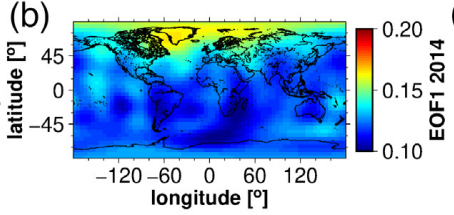

(c)

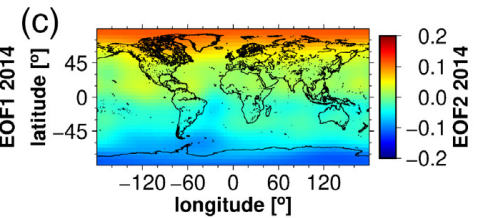

(e)

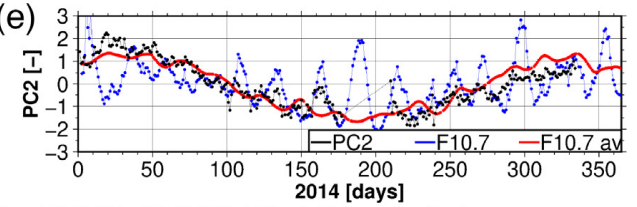

Corrected Density $(s, t)=(A(s)+B(s) D(t)+C(s) E(t)) *$ Model Density $(s, t)$

Figure 8. Temporal mean (a), and PCA derived spatial (b,c) and temporal (d,e) variability of empirical density corrections in 2014.

Table 4. Details of the ANN models and and their evaluation criteria for $7 \mathrm{~d}$ prediction.

\begin{tabular}{lccccccc}
\hline Method & Year & N.O.D & N.O.L & N.O.H & MSE & SNR \\
\hline NLNNR & 2003 & 90 & 16 & 3 & 0.0007 & 29.3498 \\
NAR & 2004 & 94 & 18 & 14 & 0.0086 & 18.4994 \\
& 2003 & 69 & 2 & 3 & 0.0015 & 25.9074 \\
NARX & 2004 & 65 & 5 & 13 & 0.0144 & 16.2756 \\
& 2003 & 90 & 15 & 7 & 0.0011 & 27.0834 & 0.89956 \\
& 2004 & 90 & 15 & 7 & 0.0035 & 22.3906 \\
\hline
\end{tabular}

Table 5. Details of the ANN models and and their evaluation criteria for $14 \mathrm{~d}$ prediction.

\begin{tabular}{lccccccc}
\hline Method & Year & N.O.D & N.O.L & N.O.H & MSE & SNR \\
\hline NLNNR & 2003 & 90 & 2 & 3 & 0.0025 & 23.6268 \\
\multirow{2}{*}{ NAR } & 2004 & 90 & 5 & 14 & 0.0047 & 21.3115 \\
& 2003 & 62 & 10 & 8 & 0.0050 & 20.5443 \\
NARX & 2004 & 64 & 16 & 4 & 0.0543 & 0.8923 \\
& 2003 & 90 & 5 & 5 & 0.0014 & 26.7186 \\
\\
\hline
\end{tabular}

Table 6. Details of the ANN models and and their evaluation criteria for $30 \mathrm{~d}$ prediction.

\begin{tabular}{lccccccc}
\hline Method & Year & N.O.D & N.O.L & N.O.H & MSE & SNR \\
\hline NLNNR & 2003 & 58 & 2 & 3 & 0.0106 & 17.2804 \\
\multirow{2}{*}{ NAR } & 2004 & 55 & 5 & 7 & 0.0089 & 18.9332 \\
\multirow{2}{*}{ NARX } & 2003 & 73 & 3 & 12 & 0.0317 & 12.5445 \\
& 2004 & 69 & 15 & 7 & 0.0237 & 14.6936 \\
\\
\hline
\end{tabular}


Table 7. Details of the ANN models and and their evaluation criteria for $90 \mathrm{~d}$ prediction.

\begin{tabular}{lccccccc}
\hline Method & Year & N.O.D & N.O.L & N.O.H & MSE & SNR \\
\hline NLNNR & 2003 & 67 & 2 & 2 & 0.0125 & 17.343 \\
NAR & 2004 & 62 & 5 & 2 & 0.0149 & 16.6799 \\
& 2003 & 69 & 2 & 4 & 0.0465 & 11.649 \\
NARX & 2004 & 66 & 16 & 3 & 0.0123 & 17.5070 & 0.7785 \\
& 2003 & 90 & 11 & 5 & 0.0121 & 0.872 \\
& 2004 & 90 & 11 & 13 & 0.0060 & 20.6048 \\
\hline
\end{tabular}
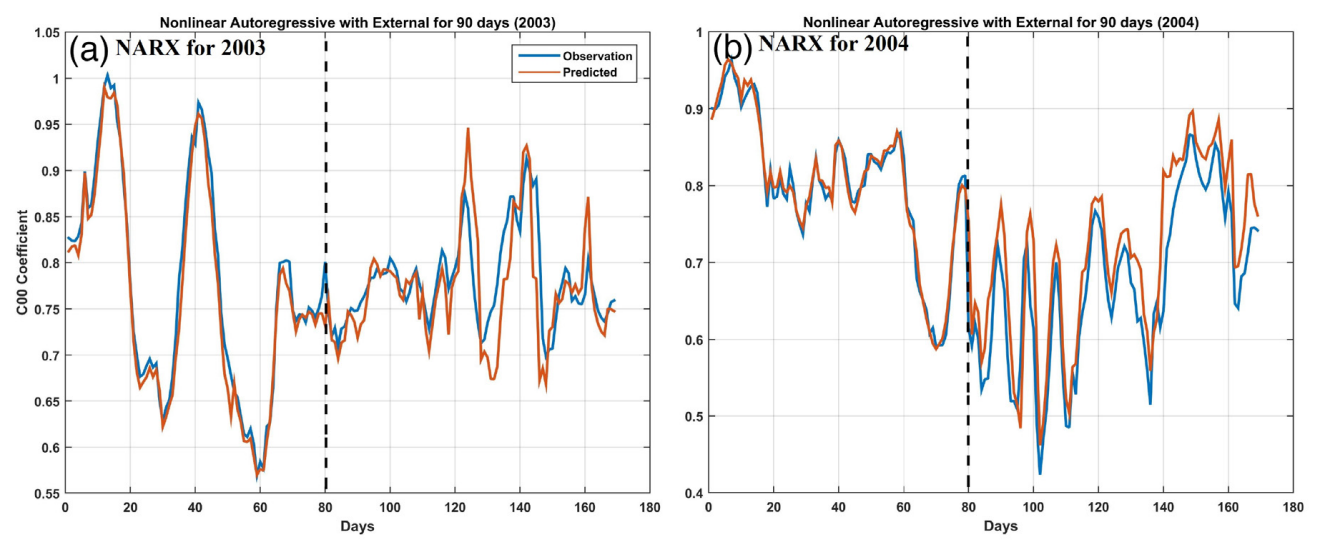

Figure 9. Comparison between the NARX model results and daily scales for $C_{00} 90 \mathrm{~d}$ prediction, where (a) corresponds to the year 2003 and (b) to 2004 .

statistics using the metrics of eqs (13), (14) and (15). Fig. 1 shows the flowchart of the ANN, which is implemented in this study. Numerical results of this paper are discussed in Section 3.4.

\section{RESULTS}

\subsection{Uncertainties of the accelerometer derived thermospheric neutral density}

Fig. 2 shows the effect of the individual uncertainties, as well as the combined effect on the accelerometer derived density for 1 January 2004 and 1 January 2014. In 2004, the observed nongravitational forces appear to greatly impact on the quality of the GRACE derived densities, followed by the force coefficient and the relative velocity. In 2014, it shows an opposite effect, but in general, the density uncertainties are greater in 2014 than for 2004. This could be because of the higher solar activities in 2014 (Masutti et al. 2016). This can also be seen in Fig. 3, where the mean uncertainty of the density profiles is shown for each month and each component. The differences from month to month are found to be smaller than the differences between the different sources of uncertainty.

To simplify the comparison between 2004 and 2014, Fig. 4 shows the mean uncertainties of densities in 2004 and 2014. The main differences can be seen in the impact of the mass $m$, the relative velocity $v_{\text {rel }}$ and the force coefficient $C_{\mathrm{a}}$. This is because the partial derivatives $\left(\frac{\partial \rho}{\partial a_{\mathrm{obs}, \mathrm{x}}}, \frac{\partial \rho}{\partial a_{\mathrm{SRP}, \mathrm{x}}}\right.$ and $\left.\frac{\partial \rho}{\partial a_{\mathrm{ERP}, \mathrm{x}}}\right)$ do not contain the acceleration term, which changes considerably from 2004 to 2014. Overall, by considering the quality of $a_{o b s}, a_{\mathrm{SRP}}$ and $a_{\mathrm{ERP}}$, the uncertainty of GRACE derived densities is found to be similar in 2004 and 2014.

Finally, we analyze the signal to noise ratio (SNR, eq. 14). Table 2 summarizes the results in 2014. It shows that the magnitude is higher than that of 2004. In general, SNR is found to be higher than one for 94.0 per cent of estimates in 2004 and for 100.0 per cent in 2014 . We found that 97.4 per cent of the points in 2014 have a SNR of higher than 10 indicating that the quality of GRACE derived neutral density is good and the along-track estimates can be used to correct model simulations. It is worth mentioning here that the quality of density estimations in 2014 is better than 2004, which might be related to the stronger amplitude of density changes in 2014 (compared to 2004).

\subsection{Impact of the GRACE derived corrections on orbit determination applications}

In Section 3.1, we showed that the quality of GRACE derived thermospheric neutral densities is acceptable. Here, we assess the impact of these estimates on LEO orbit determination application. It is well known that the neutral density of atmosphere affects the satellite's motion (details can be found in Seeber 2003; Xu et al. 2011, and Appendix B). Orbit integration is applied for $10 \mathrm{~d}$ of a CHAMPlike orbit (which means orbital parameters are similar to those of CHAMP) but at altitudes of 200, 400 and $600 \mathrm{~km}$ (orbital properties are reported in Table 3). Numerical implementation is based on an explicit Runge-Kutta approach with the error control of about $10^{-16}$ and automated step size (Shampine \& Reichelt 1997). The initial conditions for dynamic orbit propagation are listed in Table 3. To simulate the orbits, EGM96 (up to degree and order 70) is applied to account for the gravity field of the Earth. Ephemerids of the Moon and Sun are respectively calculated using the theory ELP-2000/82 represented by Chapront-Touze and Chapront and analytical formulas (Chapront-Touzé \& Chapront 1983). The solar and Earth radiation pressure are obtained from the method in Vielberg et al. (2018), and the International Earth Rotation and Reference Systems Service (IERS) formulations are used for solid Earth tide modelling (Vondrák \& Richter 2004). Within the orbit integration, drag forces are computed at the altitude of satellite orbits using densities derived from the original NRLMSISE-00 model and the scaled version. This means that the differences in the estimated 
NAR

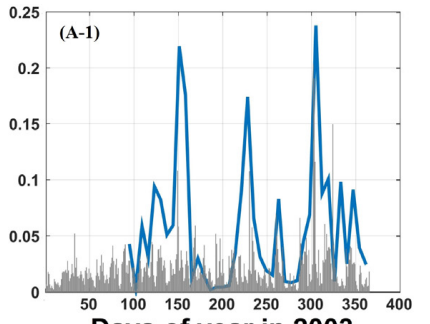

Days of year in 2003

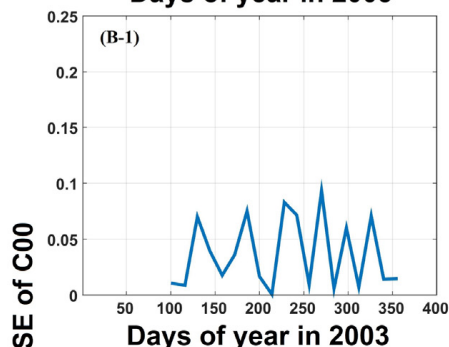

岕

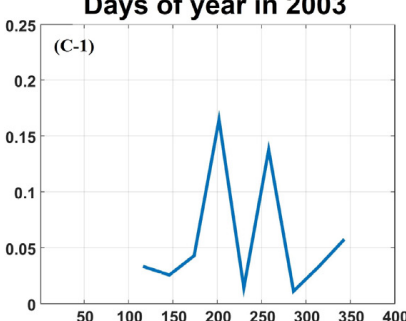

Days of year in 2003

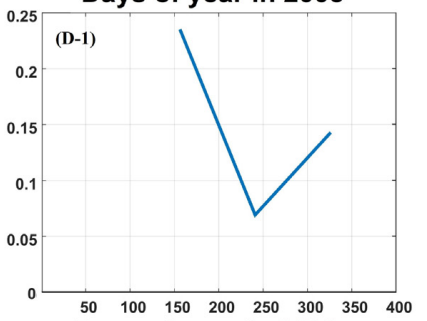

Days of year in 2003

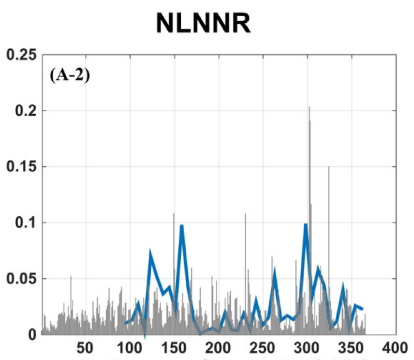

Days of year in 2003

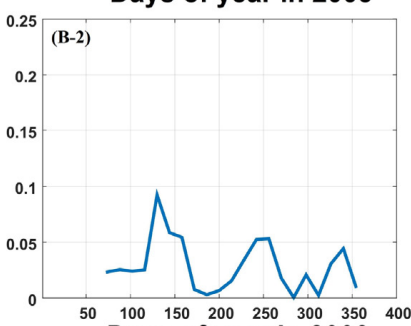

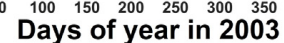

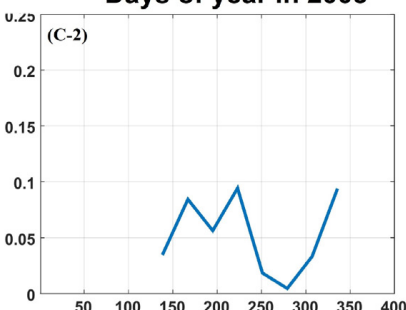

Days of year in 2003

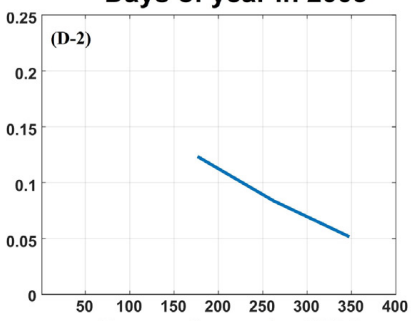

Days of year in 2003

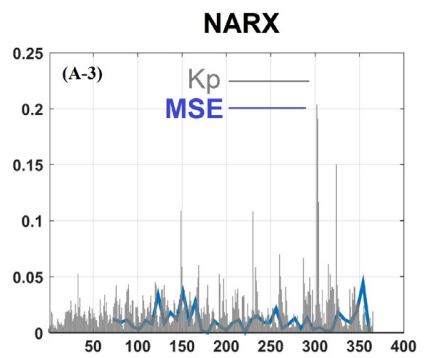

Days of year in 2003

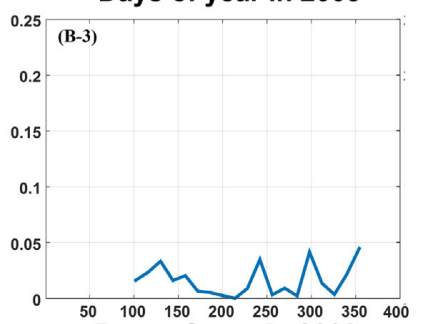

Days of year in 2003

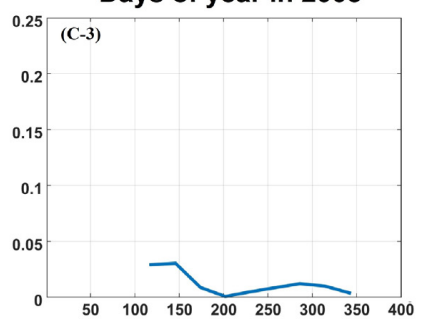

Days of year in 2003

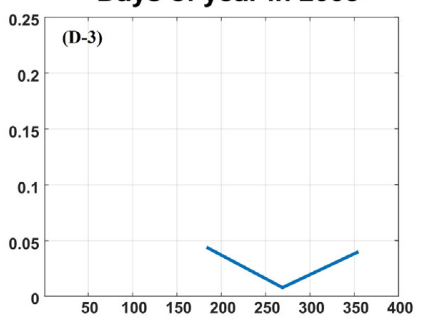

Days of year in 2003

Figure 10. Comparison of the NARX predictions of $C_{00}$ using the GRACE derived daily scales, which are computed for (a) $7 \mathrm{~d}$, (b) $14 \mathrm{~d}$, (c) $30 \mathrm{~d}$ and (d) $90 \mathrm{~d}$ of 2003. Different methods of NAR, NLNNR and NARX are shown in the first, second and third columns from left, respectively.

orbits are the result of using different neutral density values to compute the drag forces. It is worth mentioning that selecting different background models such as the reference gravity model to preform simulations does not alter our main conclusion, because their impact cancels out when computing differences between the integrated orbits.

Fig. 5 shows the differences in the estimated orbits at different altitudes. The results correspond to 4-14 March 2004 and indicate that the range of errors for 600,400 and $200 \mathrm{~km}$ orbits reaches up to $\sim 0.831, \sim 2.304$ and $\sim 3.819 \mathrm{~km}$ after $10 \mathrm{~d}$, respectively.

\subsection{Spatial and temporal variability of GRACE derived empirical corrections}

In this section, we show the spatial and temporal variability of computed GRACE derived empirical corrections (from eq. 9). Spatially averaged daily empirical corrections of 2004 and 2014 are presented in Fig. 6, where values smaller than 1 indicate that the model overestimates the thermospheric neutral density, and those bigger than
1 show underestimation. During both years, one can find periods with over- and underestimations. These are found typical during medium solar activity Vielberg et al. (2018). A comparison with the $F_{10.7}$ index (scaled to be between 0.5 and 1.5) is shown as well, which indicates similarity (correlation coefficient of 0.54 and 0.68 in 2004 and 2014, respectively) in the pattern of corrections and the evolution of solar activity.

Yearly averaged mean fields of the empirical corrections for the years 2004 and 2014 are presented in Figs 7(a) and 8(a), respectively. In the year 2014, the corrections are found to vary between 0.75 and 0.90 indicating that the models overestimate the densities during higher solar activity. In comparison, the variability in 2004 is larger, where corrections are largely found to be between 0.75 and 1.05 . In both years, errors of the empirical model densities at the poles are larger than at around the equator. This pattern is likely related to the poor performance of the model at the poles (see, e.g. Fujiwara \& Miyoshi 2010). In fact, temperature observations in NRLMSISE00 are restricted in the polar area resulting in poor temperature profiles in this region and a biased estimation of neutral density. In contrast, GRACE has a good spatial coverage over the polar 

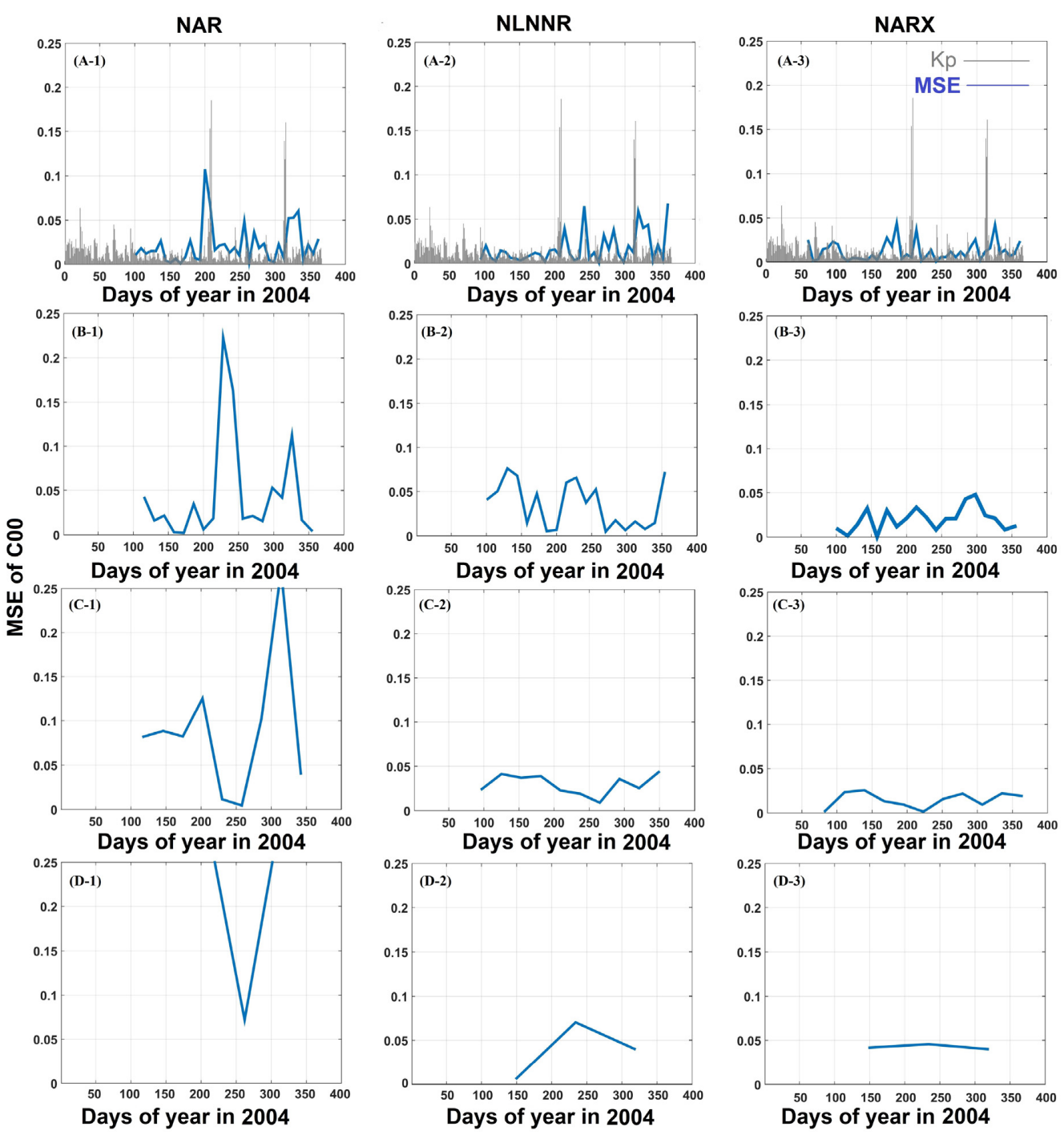

Days of year in 2004

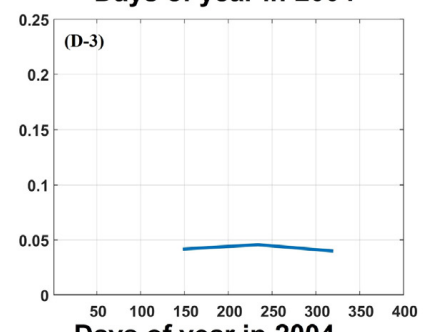

Figure 11. Comparison of the NARX predictions of $C_{00}$ using the GRACE derived daily scales, which are computed for (a) $7 \mathrm{~d}$, (b) $14 \mathrm{~d}$, (c) $30 \mathrm{~d}$ and (d) $90 \mathrm{~d}$ of 2004. Different methods of NAR, NLNNR and NARX are shown in the first, second and third columns from left, respectively.

area, and therefore, the computed corrections can be accepted with confidence.

In order to provide more insights about variability of the estimated daily correction, Principal Component Analysis (PCA, Forootan 2014, chapter 3) is applied to extract their dominant modes. Temporal averages of the year 2004 and 2014 are shown in Figs 7(a) and $8(\mathrm{a})$, while the spatially orthogonal components of PCA are shown in Figs $7(\mathrm{~b}, \mathrm{c})$ and $8(\mathrm{~b}, \mathrm{c})$ and their corresponding uncorrelated temporal patterns can be found in Figs $7(\mathrm{~d}, \mathrm{e})$ and $8(\mathrm{~d}, \mathrm{e})$. The first two in 2004 represent 63 and 18 per cent of the total variance, and in 2014, 70 and 16 per cent, respectively. The dipole patterns with a yearly cycle in the second mode in 2004 (and in the first mode in 2004) indicate the overestimation of NRLMISISE-00 during the summer time and its underestimation during winter. The second mode (panels c and e in Figs 7 and 8) likely correspond to longitudinal (upwards propagating) waves. Strong correlations are also found between the daily averaged $F_{10.7}$ index and the first mode of 2014, as well as the second mode of 2004. This indicates that the skill of NRLMSISE-00 varies spatially in time. Since the corrections represent strong correspondence to the solar activity and also due to the model's weak over polar regions, we suggest to correct the model's densities using the dominant PCA modes as shown in Figs 7 and 8 based on $\rho(s, t)_{\text {corrected model }}=A(s)+(B(s) D(t)$ $+C(s) E(t)) \rho(s, t)_{\text {original model }}$.

\subsection{Predicting daily scales using ANN techniques}

In the previous section, we showed that the daily empirical corrections are beneficial for NRLMSISE-00. Here, we assess whether data driven techniques can be used to predict these corrections when there is no GRACE observations available. Following Section 2.3, the training scenarios for the ANN methods of NLNNR, NAR and NARX are numerically implemented by examining the daily scales of the entire year 2003 and 2004. We select 2003 instead of 2014 to emphasize that this implementation is not restricted to a specific period. Target prediction periods are selected at 7, 14,30 and $90 \mathrm{~d}$ from the current time of the system. This means that we intend to predict corrections for thermospheric models up to seasonal timescales.

In fact, selecting the structure of ANNs has a direct impact on the accuracy of prediction models during the training and prediction periods. For example, the number of hidden neurons can influence the magnitude of errors projected on the nodes (which are used to 
Table 8. Comparison of errors in the prediction of daily corrections over the years 2003 and 2004.

\begin{tabular}{lccc}
\hline $\begin{array}{l}\text { Prediction } \\
\text { Length }\end{array}$ & Method & $\begin{array}{c}\text { Median of MSEs } \\
\text { of MSEs }\end{array}$ & $\begin{array}{c}\text { Standard deviations } \\
\text { of MSEs }\end{array}$ \\
\hline 2003: $7 \mathrm{~d}$ & NLNNR & 0.0186 & 0.0244 \\
2003: $7 \mathrm{~d}$ & NARX & 0.0094 & 0.0098 \\
2003: $7 \mathrm{~d}$ & NAR & 0.0396 & 0.0590 \\
2003: $14 \mathrm{~d}$ & NLNNR & 0.0240 & 0.0233 \\
2003: $14 \mathrm{~d}$ & NARX & 0.0137 & 0.0139 \\
2003: $14 \mathrm{~d}$ & NAR & 0.0176 & 0.0316 \\
2003: $30 \mathrm{~d}$ & NLNNR & 0.0461 & 0.0348 \\
2003: $30 \mathrm{~d}$ & NARX & 0.0087 & 0.0106 \\
2003: $30 \mathrm{~d}$ & NAR & 0.0329 & 0.0552 \\
2003: $90 \mathrm{~d}$ & NLNNR & 0.0838 & 0.0353 \\
2003: $90 \mathrm{~d}$ & NARX & 0.0395 & 0.0194 \\
2003: $90 \mathrm{~d}$ & NAR & 0.1419 & 0.0825 \\
2004: $7 \mathrm{~d}$ & NLNNR & 0.0112 & 0.0177 \\
2004: $7 \mathrm{~d}$ & NARX & 0.0090 & 0.0108 \\
2004: $7 \mathrm{~d}$ & NAR & 0.0154 & 0.0223 \\
2004: $14 \mathrm{~d}$ & NLNNR & 0.0374 & 0.0259 \\
2004: $14 \mathrm{~d}$ & NARX & 0.0209 & 0.0131 \\
2004: $14 \mathrm{~d}$ & NAR & 0.0198 & 0.0605 \\
2004: $30 \mathrm{~d}$ & NLNNR & 0.0305 & 0.0113 \\
2004: $30 \mathrm{~d}$ & NARX & 0.0158 & 0.0085 \\
2004: $30 \mathrm{~d}$ & NAR & 0.0822 & 0.0792 \\
2004: $90 \mathrm{~d}$ & NLNNR & 0.0403 & 0.0317 \\
2004: $90 \mathrm{~d}$ & NARX & 0.0418 & 0.0029 \\
2004: $90 \mathrm{~d}$ & NAR & 0.4241 & 0.2116 \\
\hline
\end{tabular}

generate the outputs), and therefore, the output's uncertainties are influenced. In other words, the excessive hidden neurons unrealistically increase the complexity of the problem and lead to biases in outputs. This also significantly deviates the capability of ANNs in the prediction period (see e.g. Panchal et al. 2011). Therefore, an empirically derived rule-of-thumb is considered to select the optimal size of the hidden layer in ANNs, which is chosen to be between the size of the input and size of the output layers $\mathrm{Xu} \&$ Chen (2008). This is numerically tested by changing the number of layers between 1 and 15. Finally, the length of delays is selected to be between 0 and 20. Structures of the ANNs with the best statistics in the above evaluation criteria are reported in Tables 4 , 5, 6 and 7, where the columns 3,4 and 5 indicate the optimum ANN parameters that correspond to the lowest MSE values for the number of data (N.O.D) for training, the number of lag (N.O.L), and the number of hidden Layer(s) (N.O.H), respectively. The results are computed for predictions of 7, 14, 30 and $90 \mathrm{~d}$. Performance criteria in columns 6,7 and 8 of these tables indicate that the quality of ANN predictions is acceptable for forecast of up to $90 \mathrm{~d}$.

From Tables 4 to 7 , it is clear that the NARX model provides the best statistical performance to predict daily scales for 7, 14, 30 and $90 \mathrm{~d}$. Therefore, this model is selected to discuss the details of training and validation steps. This however does not mean that the other models are not suitable for prediction. In fact, when only daily scales or only indices are available, respectively, NLNNR and NAR are equally recommended to be used.

As an example, results of the NARX's prediction for $90 \mathrm{~d}$ are shown in Fig. 9. The $C_{00}$ coefficients of the daily scales are shown that represent the biggest part of the variance in daily scale fields. A visual investigation indicates that although evolution of the daily corrections is complicated and non-linear, NARX is able to perform an acceptable prediction (i.e. a correlation coefficient of 0.92 between the NARX prediction and the GRACE derived $C_{00}$ ).
We also validate the performance of NARX for the entire 2003 and 2004. For this $C_{00}$ of the daily scales is predicted in moving windows of 7, 14, 30 and $90 \mathrm{~d}$ covering the entire years. MSEs are computed and plotted against time (see Figs 10 and 11), which indicate that the magnitude of errors increases from 7 to $90 \mathrm{~d}$. Median and standard deviations of the errors are reported in Table 8 indicating that the maximum of the median error (in predicting $C_{00}$ ) reaches to 0.04 (i.e. equivalent with $\sim 0.125$ in terms of scales). Similarity of the results in 2003 and 2004 indicates that the ANN's prediction performance is relatively stable. It should be mentioned here that during the validation phase, we used scales that were not used in the training of ANNs.

\section{CONCLUSION}

In this study, the GRACE derived accelerometer measurements are used to estimate thermospheric neutral density at the altitude of GRACE satellites. Uncertainties influencing these estimates are evaluated here and we showed that they are in acceptable range (see SNRs in Table 2). Next, daily scales are computed from the GRACE derived density estimates and those derived from NRLMSISE-00. These scales are interpreted here as empirical corrections to be applied on model simulations. The impact of these corrections is then evaluated for a range of orbits covering the altitude of 200, 400 and $600 \mathrm{~km}$, to show that the derived corrections are suitable for orbit determination of LEO satellites. Spatial and temporal variability of the corrections are explored by applying PCA on selected years of 2004 and 2014. Finally, various ANN prediction techniques are evaluated to predict these corrections for 7, 14, 30 and $90 \mathrm{~d}$. The following summarizes the major outcomes of the study.

(1) Previous studies, such as Mehta et al. (2017a) and Vielberg et al. (2018), showed that the GRACE derived thermospheric neutral densities are generally more reliable model outputs, especially on short timescales and during strong solar events, for example during 
the St. Patrick's Day storm on 17 March 2015. Here, we showed that, even on the seasonal to yearly timescales, neutral densities estimated from GRACE observations represent high correspondence to the solar (and geomagnetic activities), for example see Figs 6-8. These results confirm that the computed daily corrections are sensible.

(2) Application of PCA on the estimated daily corrections indicate that the total neutral density simulated by NRLMSISE- 00 does not have a constant quality over the globe, and for example its quality is worse over the polar regions compared to those over the equator.

(3) Applying the scaled densities on orbit determination experiments show an error of $0.831,2.304$ and $3.819 \mathrm{~km}$ after $10 \mathrm{~d}$ for CHAMP-like orbits at altitudes of 200, 400 and $600 \mathrm{~km}$, respectively. These errors are considerable and need to be taken into account in related applications.

(4) ANN technique is efficient for predicting GRACE derived daily empirical corrections up to $90 \mathrm{~d}$. The maximum magnitude of the median errors is $\sim 0.125$ in terms of scales. In real applications, the original model derived neutral density estimations can be replaced with scaled values and be used for example drag estimation within orbit determination procedures or for re-entry predictions.

The empirical corrections derived from this study are computed by averaging all accelerometer observations of $1 \mathrm{~d}$. In future, the results will be compared with enhanced model simulations through merging of GRACE corrections and model outputs in a data assimilation system.

\section{ACKNOWLEDGEMENTS}

The authors are grateful to the A.E. Dr Louise Alexander, Professor Kosuke Heki , and anonymous reviewers for providing useful comments, which helped us to improve the quality of this study. E. Forootan, K. Vielberg and C. Lück would like to acknowledge the research grant from the German Aerospace Center (DLR, D-SAT

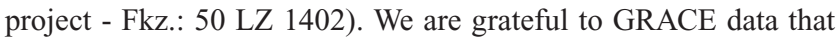
were freely available from JPL. ${ }^{1}$

\section{REFER EN CES}

Albertella, A., Migliaccio, F. \& Sanso', F., 2002. GOCE: the Earth gravity field by space gradiometry, Celest. Mech. Dyn. Astron., 83(1), 1-15.

Belehaki, A., Stanislawska, I. \& Lilensten, J., 2009. An overview of ionosphere-thermosphere models available for space weather purposes, J. Space Sci. Rev., 147, 271-313.

Bettadpur, S., 2012. Gravity Recovery and Climate Experiment, product specification document, Center for Space Research, The University of Texas at Austin.

Bilitza, D., 2000. The importance of euv indices for the international reference ionosphere, Phys. Chem. Earth., Part C, 25(5-6), 515-521.

Bowman, B.R., Tobiska, W.K., Marcos, F.A., Huang, C.Y., Lin, C.S. \& Burke, W.J., 2008. A new empirical thermospheric density model JB2008 using new solar and geomagnetic indices, in AIAA/AAS Astrodynamics Specialist Conference and Exhibit, Honolulu, Hawaii.

Bruinsma, S., 2013. GOCE + Theme 3: Air density and wind retrieval using GOCE data: validation report: ESA AO/1-6367/10/NL/AF, Tech. Rep. Version 1.1-3 June 2013.

Bruinsma, S. \& Biancale, R., 2003a. Total densities derived from accelerometer data, J. Spacecraft Rockets, 40(2), 230-236.

Bruinsma, S., Thuillier, G. \& Barlier, F., 2003. The DTM-2000 empirical thermosphere model with new data assimilation and constraints at lower

${ }^{1} \mathrm{ftp} / / /$ podaac-ftp.jpl.nasa.gov/allData/grace/L1B/JPL/RL02/ boundary: accuracy and properties, J. Atmos. Solar-Terrest. Phys., 65(9), 1053-1070.

Bruinsma, S., Tamagnan, D. \& Biancale, R., 2004. Atmospheric densities derived from CHAMP/STAR accelerometer observations, Planet. Space Sci., 52(4), 297-312.

Chakraverty, S. \& Mall, S., 2017. Artificial Neural Networks for Engineers and Scientists: Solving Ordinary Differential Equations, CRC Press.

Chan, K.Y., Dillon, T.S., Singh, J. \& Chang, E., 2012. Neural-network-based models for short-term traffic flow forecasting using a hybrid exponential smoothing and levenberg-marquardt algorithm, IEEE Trans. Intell. Transport. Syst., 13(2), 644-654.

Chapront-Touzé, M. \& Chapront, J., 1983. The lunar ephemeris elp 2000, Astron. Astrophys., 124, 50-62.

Chen, S. \& Billings, S., 1991. Neural networks for nonlinear dynamic system modelling and identification, Res. Report. Acse Report. Dept of Automatic Control and System Engineering. University of Sheffield, 436.

Choury, A., Bruinsma, S. \& Schaeffer, P., 2013. Neural networks to predict exosphere temperature corrections, Space Weather, 11(10), 592-602.

Codrescu, M., Fuller-Rowell, T. \& Minter, C., 2004. An ensemble-type kalman fillter for neutral thermospheric composition during geomagnetic storms, Space Weather, 2, S11002.

Codrescu, S., Codrescu, M. \& Fedrizzi, M., 2018. An ensemble kalman filter for the thermosphere-ionosphere, Space Weather, 16(1), 57-68.

Doornbos, E., 2011. Thermospheric Density and Wind Determination From Satellite Dynamics, Springer Berlin Heidelberg.

Doornbos, E. et al., 2009. Air density models from multiple-satellite drag observations, DEOS/TU Delft Scient. Rep., 1.

Flury, J., Bettadpur, S. \& Tapley, B., 2008. Precise accelerometry onboard the grace gravity field satellite mission, Adv. Space Res., 42, 1414-1423.

Forootan, E., 2014, Statistical signal decomposition techniques for analyzing time-variable satellite gravimetry data, $P h D$ thesis, University of Bonn, p. 131.

Forootan, E., Didova, O., Kusche, J. \& Löcher, A., 2013. Comparisons of atmospheric data and reduction methods for the analysis of satellite gravimetry observations, J. geophys. Res.-Solid Earth, 118, 1-15.

Forootan, E. et al., 2014. Multivariate prediction of total water storage changes over west africa from multi-satellite data, Surv. Geophys., 35(4), 913-940.

Fujiwara, H. \& Miyoshi, Y., 2010. Morphological features and variations of temperature in the upper thermosphere simulated by a whole atmosphere gcm, Ann. Geophys., 28, 427-437.

Fuller-Rowell, T., Minter, C. \& Codrescu, M., 2004. Data assimilation for neutral thermospheric species during geomagnetic storms, Radio Sci., 39, RS1S03.

Jacchia, L.G., 1970. New static models of the thermosphere and exosphere with empirical temperature profiles, SAO Spec. Rep., 313.

Kisi, Ö., 2004. Multi-layer perceptrons with levenberg-marquardt training algorithm for suspended sediment concentration prediction and estimation, Hydrol. Sci. J., 49(6), 1025-1040.

Loeb, N. et al., 2018. Clouds and the earths radiant energy system (ceres) energy balanced and filled (ebaf) top-of-atmosphere (toa) edition-4.0 data product, J. Clim., 31, 895-918.

Masutti, D., March, G., Ridley, A.J. \& Thoemel, J., 2016. Effect of the solar activity variation on the global ionosphere thermosphere model (gitm), Ann. Geophys., 34(9), 725-736.

Matsuo, T., Fedrizzi, M., Fuller-Rowell, T. \& Codrescu, M., 2012. Data assimilation of thermospheric mass density, Space Weather, 10, 05002.

Matsuo, T., Lee, I.-T. \& Anderson, J., 2013. Thermospheric mass density specification using an ensemble kalman filter, J. geophys. Res. - Space Physics, 118, 1339-1350.

Mayaud, P.N., 1980. Derivation Meaning and Use of Geomagnetic Indices, Geophysical Monograph, 322, America Geophysical Union.

Mehta, P. \& Linares, R., 2018. A new transformative framework for data assimilation and calibration of physical ionosphere-thermosphere models, Space Weather, 16, 1086-1100.

Mehta, P., Walker, A., Sutton, E. \& Godinez, H., 2017a. New density estimates derived using accelerometers on-board the champ and grace satellites, Space Weather, 15, 558-576. 
Minter, C., Fuller-Rowell, T. \& Codrescu, M., 2004. Estimating the state of the thermospheric composition using Kalman filtering, Space Weather, 2, S04002.

Morozov, A., Ridley, A., Bernstein, D., Collins, N., Hoar, T. \& Anderson, J., 2013. Data assimilation and driver estimation for the global ionospherethermosphere model using the ensemble adjustment Kalman filter, $J$. Atmos. Solar-Terres. Phys., 104, 126-136.

Murray, S., Henley, E., Jackson, D. \& Bruinsma, S., 2015. Assessing the performance of thermospheric modeling with data assimilation throughout solar cycles 23 and 24, Space Weather, 13, 220-232.

Panchal, G., Ganatra, A., Kosta, Y. \& Panchal, D., 2011. Review on methods of selecting number of hidden nodes in artificial neural network, Int. J. Comput. Theory Eng., 3(2), 332-337.

Pérez, D., Wohlberg, B., Alan Lovell, T., Shoemaker, M. \& Bevilacqua, R., 2014. Orbit-centered atmospheric density prediction using artificial neural networks, 98, 9-23.

Picone, J., Hedin, A., Drob, D.P. \& Aikin, A., 2002. NRLMSISE-00 empirical model of the atmosphere: statistical comparisons and scientific issues, J. geophys. Res.: Space Phys. (1978-2012), 107(A12), SIA-15.

Qian, L. \& Solomon, S., 2012. Thermospheric density: an overview of temporal and spatial variations, Space. Sci. Rev., 168(1-4), 147-173.

Reigber, C., Lühr, H. \& Schwintzer, P., 2002. CHAMP mission status, $A d v$. Space Res., 30(2), 129-134.

Roble, R. \& Ridley, E., 1994. A thermosphere-ionosphere-mesosphereelectrodynamics general circulation model (time- $\mathrm{gcm}$ ): equinox solar cycle minimum simulations (30-500 km), Geophys. Res. Lett., 21, 417-420.

Seeber, G., 2003. Satellite Geodesy: Foundations, Methods, and Applications, Walter de Gruyter.

Shampine, L.F. \& Reichelt, M.W., 1997. The MATLAB ODE suite, SIAM J. Sci. Comput., 18(1), 1-22.

Storz, M., Bowman, B., Branson, J., Casali, S. \& Tobiska, W., 2005. High accuracy satellite drag model (hasdm), Adv. Space Res., 36(12), 24972505.

Sutton, E., 2008, Effects of solar disturbances on the thermosphere densities and winds from CHAMP and GRACE satellite accelerometer data, $P h D$ thesis, University of Colorado, Boulder.

Sutton, E., 2018. A new method of physics-based data assimilation for the quiet and disturbed thermosphere, Space Weather, 16(6), 736-753.

Tapley, B.D., Bettadpur, S., Watkins, M. \& Reigber, C., 2004. The gravity recovery and climate experiment: mission overview and early results, Geophys. Res. Lett., 31(9), doi:10.1029/2004GL019920.

Vallado, D.A. \& Finkleman, D., 2014. A critical assessment of satellite drag and atmospheric density modeling, Acta Astron., 95, 141-165.

Vielberg, K., Forootan, E., Lück, C., Löcher, A., Kusche, J. \& Börger, K., 2018. Comparison of accelerometer data calibration methods used in thermospheric neutral density estimation, Ann. Geophys., 36(3), 761-779.

Vondrák, J. \& Richter, B., 2004. International earth rotation and reference systems service (iers, www.iers.org), J. Geod., 77(10-11), 585-678.

Xu, G., Tianhe, X., Chen, W. \& Yeh, T.-K., 2011. Analytical solution of a satellite orbit disturbed by atmospheric drag, Mon. Not. R. Astron. Soc., 410(1), 654-662.

Xu, J.,Wang, W Zhang, S., Liu, X. \& Yuan, W., 2015. Multiday thermospheric density oscillations associated with variations in solar radiation and geomagnetic activity, J. geophys. Res.: Space Phys., 120, 3829-3846.

Xu, S. \& Chen, L., 2008. A novel approach for determining the optimal number of hidden layer neurons for fnns and its application in data mining, in Proceedings of the 5th International Conference on Information Technology and Applications (ICITA 2008), pp. 23-26, June, Cairns, Queensland, Australia.

\section{A P PENDIX A}

Fig. A1(a) shows a global thermospheric neutral density map derived from NRLMSISE-00 on 1 February 2004 at 18:00 hr. We convert GRACE derived accelerometer observations of the same date to along-track density profiles and compute scales. Results of this conversion are shown in Fig. A1(b). Finally, these scales are (a) Original Model NRLMSISE-00

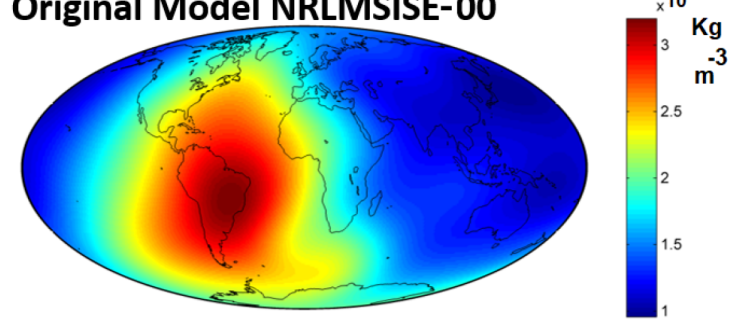

(b) Scales, i.e. GRACE/NRLMSISE-00

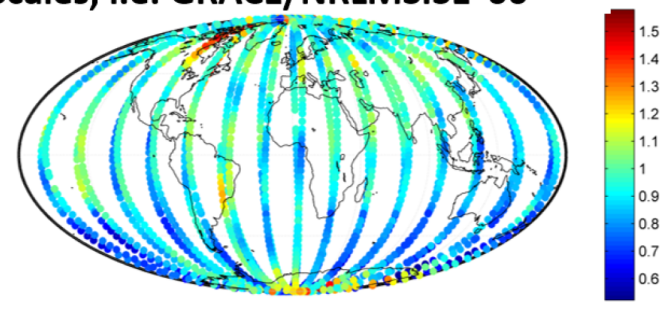

(c) NRLMSISE-00 after scaling

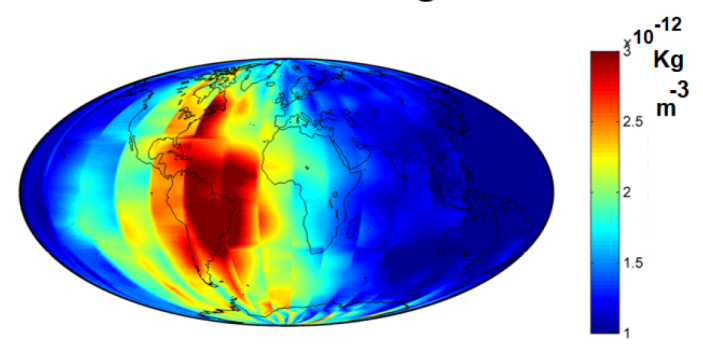

Figure A1. (a) Neutral density map from NRLMSISE-00 on 1 February 2004 at 18:00 hr, (b) along track scales derived as fraction of the GRACE derived neutral density estimations and the corresponding simulations of NRLMSISE-00 and (c) corrected NRLMSISE-00 field derived by multiplying the expanded scales by the map in (a).

expanded to the spherical domain and synthesized back to grid domain following eq. (9). Corrected neutral density field is derived by multiplying the synthesized map of Fig. A1(b) by the original density field Fig. A1(a) as are shown in Fig. A1(c).

\section{APPENDIX B}

The acceleration of a satellite $(\ddot{\vec{r}})$ can be modelled by considering radial gravitational $\left(\vec{\gamma}_{r}\right)$ and perturbing $\left(\vec{\gamma}_{p}\right)$ forcing acting on its surface, which can be written as

$\ddot{\vec{r}}=\vec{\gamma}_{r}+\vec{\gamma}_{p}$.

The latter term can be formulated as

$\vec{\gamma}_{p}=\ddot{\vec{r}}_{E}+\ddot{\vec{r}}_{n}+\ddot{\vec{r}}_{\text {tides }}+\ddot{\vec{r}}_{d r a g}+\ddot{\vec{r}}_{S R}+\ddot{\vec{r}}_{a}$,

where, $\ddot{\vec{r}}{ }_{E}$ represents accelerations due to the non-spherically and inhomogeneous mass distribution within Earth (central body), and other terms are $\ddot{\vec{r}}_{n}$ : accelerations due to other celestial bodies (Sun, Moon and planets), $\ddot{\vec{r}}_{\text {tides }}$ : accelerations due to Earth and oceanic tides, $\ddot{\vec{r}}_{\text {drag }}$ : accelerations due to atmospheric drag, $\ddot{\vec{r}}_{S R}$ : accelerations due to direct solar radiation pressure, and $\ddot{\vec{r}}_{a}$ : accelerations due to Earth-reflected solar radiation pressure.

Orbit integration generally is a process of determining position in the knowledge of accelerations of eq. (B1). This integration is 
computed numerically, where all forces are explicitly calculated, and they are used as starting conditions for a step-wise integration. For low-orbiting satellites the most important non-gravitational perturbation is caused by the drag acceleration, due to the interaction between the satellite and particles of the atmosphere, hence, the appropriate mathematical modelling of the resulting forces turns out to be a rather complicated problem. Drag acceleration can be modelled as (Seeber 2003):

$\ddot{\vec{r}}_{\text {drag }}=-\frac{1}{2} \frac{c_{D} A}{m} \rho \vec{v}_{r}\left|\vec{v}_{r}\right|$, with

$\vec{v}_{r}=\left(\overrightarrow{\dot{r}}-\overrightarrow{\dot{r}}_{a}\right)$, where $m$ is the mass of satellite, $A$ is the effective cross-sectional area of the satellite, $c_{D}$ stands for the drag coefficient (satellite specific), $\rho$ indicates neutral density of the atmosphere near the satellite, and finally, $\overrightarrow{\dot{r}}$ is the velocity vector of satellite with respect to the $\vec{r}_{a}$ that is the velocity of atmosphere near the satellite. Errors in modelling drag will be reflected in eq. (B2) and finally causes errors in the orbit integration as shown in Fig. 5. 